A N N A L E S Annales de Bretagne et des Pays de l'Ouest

Anjou. Maine. Poitou-Charente. Touraine

$120-2 \mid 2013$

Face aux risques maritimes

\title{
La pluriactivité des marins du long cours au XVIIIe siècle.
}

Les gens de mer de la Seudre : du chai à l'atelier

Long-distance sailors' pluriactivity in the eighteenth century: the seamen of the Seudre, from the wine-warehouse to the workshop

\section{Thierry Sauzeau}

\section{(2) OpenEdition}

Journals

Édition électronique

URL : http://journals.openedition.org/abpo/2618

DOI : $10.4000 / a b p o .2618$

ISBN : 978-2-7535-2843-7

ISSN : 2108-6443

Éditeur

Presses universitaires de Rennes

Édition imprimée

Date de publication : 30 juin 2013

Pagination : 65-78

ISBN : 978-2-7535-2838-3

ISSN : 0399-0826

Référence électronique

Thierry Sauzeau, "La pluriactivité des marins du long cours au XVIIIe siècle. », Annales de Bretagne et des Pays de l'Ouest [En ligne], 120-2 | 2013, mis en ligne le 30 juin 2015, consulté le 19 avril 2019. URL http://journals.openedition.org/abpo/2618; DOI : 10.4000/abpo.2618 


\title{
La pluriactivité des marins au long cours au XVIII ${ }^{\mathrm{e}}$ siècle
}

\author{
Les gens de mer de la Seudre : du chai à l'atelier
}

\author{
Thierry SAUZEAU \\ Professeur d'histoire moderne, \\ CRiHAM-EA4270 - Université de Poitiers
}

Dès les années 1980, les historiens ruralistes ${ }^{1}$ ont affirmé que la pluriactivité l'emportait sur l'exercice exclusif d'un métier dans les campagnes. Le colloque organisé en 2002 à Lorient et publié en $2004^{2}$ a exploré les contours d'une pluriactivité " littorale ", définie par Gérard Le Bouëdec, dans un article publié dans les Annales de Bretagne et des Pays de l'Ouest ${ }^{3}$. Chez les marins saintongeais du grand commerce, à l'apogée de la marine à voile, l'exercice de plusieurs activités au sein d'un même foyer peut être mis en évidence grâce aux inventaires après décès. Certes, il s'agissait là d'un acte assez onéreux, donc peu accessible aux plus humbles ${ }^{4}$, mais le poids de la mortalité " océane ", qui fauchait un homme sur deux avant l'âge de 35 ans, lisse un peu ce défaut structurel ${ }^{5}$. Ce risque majeur était à l'origine de stratégies de résilience, dont la maîtrise de plusieurs savoir-faire et l'exercice domestique de plusieurs activités était le fondement. Parmi les 85 inventaires qui servent de base à cette étude, ce sont finalement 21 actes instrumentés chez des capitaines ou des officiers de marine qui peuvent être confrontés à 28 actes concernant des gens de la maistrance,

1. La Pluriactivité dans les familles agricoles, Actes du colloque de l'Association des ruralistes français (Isle-d'Abeau, 1981), Paris, ARF, 1984.

2. Le Boü̈DEc, Gérard, Ploux, François, CERINo, Christophe, GeisTDOERFER, Aliette (dir.), Entre terre et mer. Sociétés littorales et pluriactivité ( $X V^{e}-X X^{e}$ siècles), Rennes, PUR, 2004.

3. LE BoUËDEC, Gérard, "La pluriactivité dans les sociétés littorales (XVII'-XVIII" siècles) ", Annales de Bretagne et des pays de l'Ouest, $\mathrm{t} .109, \mathrm{n}^{\circ}$ 1, 2002, p. 61-90.

4. Selon Daniel Roche, le coût d'un tel acte se montait à 15 ou 20 livres au début du siècle et jusqu'à 30 ou 40 livres vers 1780, soit plus de 20 journées de travail (Le Peuple de Paris, essai sur la culture populaire au XVIII ${ }^{e}$ siècle, Paris, Aubier, 1981, p. 28).

5 . Entre le tiers et la moitié des couples n'atteignait pas 10 ans de vie commune, contre le quart pour l'ensemble de la population française, selon CABANTOus, Alain, Les Citoyens du large. Les identités maritimes en France (XVII -XIXe siècles), Paris, Aubier, 1995, p. 123. 
des maîtres de barque ou des charpentiers, tandis que 36 inventaires donnent la vision du monde des plus humbles (tonneliers et cordiers, matelots et mariniers, pêcheurs). On se propose d'y rechercher les traces d'un capital de production (outils, engins, matériaux) et d'éventuels stocks (céréales, vin, résultats de trafics ou de pillages ${ }^{6}$ ). L'objectif consiste à reconstituer l'origine des ressources des foyers maritimes, dans cette zone rurale fortement polarisée qu'était la Seudre, au XVIII ${ }^{\mathrm{e}}$ siècle. Le petit millier de gens de mer qui vivaient autour de ce modeste fleuve travaillaient essentiellement sur les flottes du long cours, à Bordeaux et La Rochelle. Dans la société de l'époque, ils se distinguaient par leur activité contractuelle et salariée ainsi que par le privilège d'appartenir aux classes de la marine (service du roi, Invalides).

Dans les deux tiers des cas, c'est l'image d'un quotidien révélé par la rupture d'une union qu'offrent nos inventaires. Décédé " en service à Rochefort ${ }^{7}$ ", François Lamoureux (Bourcefranc, 1756-1794) laissait Madeleine Brochet seule pour élever sa fille mineure, Marie ( 8 ans), dans sa petite maison du Chapus. Le veuvage était aussi synonyme de dissolution de communauté. La question est plus compliquée dans le cas d'une veuve. Lorsque le 23 novembre 1782, à son domicile sis Grand'Rüe à Marennes, décédait Marguerite Masson ${ }^{8}$, fille, veuve et mère de capitaine au long cours, à quoi correspondaient les biens inventoriés à la requête de neveux d'un second mariage? C'est là toute l'ambiguïté d'une source qui " est la plus riche et la plus concrète pour appréhender le monde de la culture matérielle ${ }^{9}$ " mais dont il faut admettre qu'elle est une addition de cas particuliers, d'histoires individuelles, de réussites ou d'échecs.

Si elle semble satisfaisante en qualité, cette source n'en pose pas moins un problème quantitatif, tant elle est difficile à débusquer. En Seudre, sur 47 notaires ayant officié durant les années 1750-1850 et dont les minutiers sont consultables aux Archives départementales de la Charente-Maritime, seuls 11 semblent avoir été dépositaires de la confiance du monde maritime. Quelques indices peuvent permettre de cibler ces officiers notariaux. À Saujon, la seule étude qui avait les faveurs des gens de mer se situait sur le port de Riberou. Dans la presqu'île d'Arvert, malgré la présence de trois à quatre notaires en activité à La Tremblade, les officiers d'Arvert avaient

6. Les menus objets détenus en grande quantité à bord par les marins du long cours, identifiés par Caroline BIDON, résultaient d'un trafic de pacotille (Effets et hardes des gens morts en mer d'après les inventaires après décès des navigants rochelais [1739-1759]), Mémoire de maîtrise, université de Poitiers, 1992, p. 117 sq.). Quant à l'économie souterraine, liée au recyclage des objets d'épave, récoltés à la laisse, elle a été analysée en détail par Alain Cabantous, Les Côtes barbares. Pilleurs d'épaves et sociétés littorales en France (1680-1830), Paris, Fayard, 1993.

7. Arch. dép. de Charente-Maritime. Inventaire du 17 mai 1794 (3E XX - 629, Me AntoineJean Gaudin, étude Ranson, Marennes).

8. Arch. dép. de Charente-Maritime. Inventaire du 16 décembre 1782 (3E XX - 377, $\mathrm{M}^{\mathrm{e}}$ Durand, étude Ranson, Marennes).

9. PARDAllHÉ-Galabrun, Annick, La Naissance de l'intime. 3000 foyers parisiens au XVII et XVIII siècles, Paris, PUF, 1988, p. 33. 
la préférence. Ils portaient des noms que l'on retrouve chez les officiers et capitaines du lieu. À côté du notaire "du quai ", la parenté jouait aussi son rôle. Pour Marennes, les notaires repérés avaient soit des parents marins, soit une charge d'officier à l'Amirauté de Marennes.

Les notaires connaissaient donc bien leurs clients et on peut visiter les maisons des gens de mer à la recherche d'indices d'une activité économique plurielle, entre terre et mer. Chez ces salariés du grand commerce, il s'agira de mettre en évidence une capacité à produire de manière autonome, dans le cadre de l'économie domestique ou bien par la participation à un échange quelconque. Cette enquête peut débuter par la structure des maisons, à la recherche de pièces spécialisées. Puis, laissant volontairement de côté le capital maritime (embarcation, engins de pêche), on soumettra chaque groupe social à un audit. Il s'agira d'estimer la pénétration du " rural " dans le quotidien des marins, par l'évaluation du capital d'exploitation agricole (outils, bétail, stocks, propriétés baillées). On s'attachera également aux indices de l'activité artisanale. Enfin, on recherchera, dans la propriété de menus objets, de coupons de tissu ou de quantités commerciales d'épices, la " frontière entre l'usage intime et la démarche lucrative ${ }^{10} "$.

\section{Du matelot au capitaine : lieux de vie, maisons pour travailler}

Au contraire de celle de l'île d'Oléron voisine, l'architecture des villages bordiers de l'estuaire de la Seudre n'a pas retenu l'attention de l'inventaire. Il est néanmoins possible de repérer nombre de déterminismes (sols, vents dominants, rareté du bois, des pierres) qui frappaient indifféremment Oléron et la Seudre. Les maisons étaient basses, la hauteur sous plafond atteignait $2 \mathrm{~m}$ à $2,20 \mathrm{~m}$, rarement plus. Elles n'avaient, le plus souvent, qu'un rez-de-chaussée; le premier étage, lorsqu'il existait, était un grenier. La description de la maison oléronnaise, dont la validité pour le XVIII ${ }^{\mathrm{e}}$ siècle a été vérifiée chez les sauniers ${ }^{11}$, sied bien aux gens de mer de la Seudre.

\section{Dépendances et annexes : de la polyvalence à la spécialisation}

Si l'architecture était à dominante horizontale, on recensait 30 demeures à étage, surtout dans les bourgs : Marennes, Bourcefranc, Riberou ou La Tremblade. Les maisons de gens de mer épousaient par leur taille le niveau social de leur occupant. La distinction entre espaces domestiques et annexes consacrées à l'activité professionnelle offre une lecture de cette réalité.

10. Les pratiques commerciales individuelles à bord des long-courriers ont été étudiées par le détail, par Caroline BIDON, Effets et hardes des gens morts en mer..., op. cit., p. 117.

11. Extrait de "L'architecture de l'île d'Oléron. Architecture rurale ", Les Cahiers d'Oléron, $\mathrm{n}^{\circ} 15$, novembre 1993, p. 14, dont les données ont été confirmées par Florence EDESA, Les Sauniers de l'île d'Oléron au XVIII siècle, mémoire de master 1 sous la direction de Jacques Péret, université de Poitiers, 2001, p. 91. 
Lorsqu'ils étaient dépendants, les marins ruraux de la Seudre se distinguaient de la plèbe littorale ${ }^{12}$ ou de leurs collègues citadins ${ }^{13}$ par un moindre poids des logements à pièce unique : $10 \%$ des cas. Leur logis se caractérisait par une grande polyvalence des pièces de vie et une rareté des annexes clairement identifiées. Aux marges de l'indépendance économique, les tonneliers vivaient dans une à deux pièces tandis que les charpentiers et autres officiers mariniers disposaient de deux à trois pièces clairement domestiques. À côté de l'habitation, ces foyers économiquement indépendants comptaient un éventail d'annexes laissant entrevoir une spécialisation domestique ou économique. Cette évolution était à mettre en rapport avec la propriété d'un capital productif qui faisait défaut aux plus humbles. Les mêmes causes produisaient les mêmes effets dans le Morbihan, où les demeures de maîtres charpentiers comptaient " 3 à 4 pièces de vie et un étage, d'où l'existence de greniers ${ }^{14}$ ". Un cran au-dessus, les capitaines et les officiers de marine possédaient quant à eux des logis spacieux. La norme s'établissait autour de trois à cinq pièces de vie. L'équilibre général des logements penchait résolument vers les espaces annexes qui s'y multipliaient.

Au total, la maison du marin de la Seudre n'offrait pas un aspect différent de celle de ses voisins paysans, artisans ou bourgeois, tant chacun s'identifiait davantage au groupe social auquel il se rattachait qu'à son occupation principale : la navigation.

\section{La chambre, polyvalente par nécessité}

Élément commun aux 77 logements visités et à toutes les strates sociales, la chambre omniprésente représentait le cœur même de l'espace habitable ${ }^{15}$. Pièce à vivre chez les ménages " à l'aise ", elle révélait souvent son caractère polyvalent chez les plus humbles. Chez André Quantin ${ }^{16}$, marinier au Breuil-du-Pas, la demeure se composait de deux chambres basses. En fait, comme chez les sauniers d'Oléron ${ }^{17}$ ce logement se composait d'une pièce de vie, à vocation domestique et d'une pièce de range-

12. Florence Edesa recense ainsi 50 pièces uniques parmi les 100 inventaires qu'elle a exploitées, Ibidem, p. 91.

13. Marina Allinckx aboutit quant à elle à $36 \%$ de pièces uniques chez les 100 marins rochelais dont elle a exploité les inventaires (Les Gens de mer rochelais (1702-1764), université de Poitiers, mémoire de maîtrise, sous la direction de Jacques Péret, 1994).

14. C'est le constat que pose Erwan DANDO, Construction navale et maîtres constructeurs de navires sur le Golfe du Morbihan de 1750 à 1789, université de Bretagne-Sud, mémoire de maîtrise, sous la direction de Gérard Le Bouëdec, 1996, p. 94.

15. PARdallhé-Galabrun, Annick, La Naissance de l'intime..., op. cit., p. 17.

16. Arch. dép. de Charente-Maritime, $3 E \mathrm{LV} / 26$, le $1^{\mathrm{er}}$ décembre 1750, par Me Dillé aîné, Saujon.

17. Suivant Florence Edesa, "lorsqu'une annexe agrandissait une demeure à pièce unique, elle abritait généralement les outils et les matériaux professionnels, ce qui devait permettre à la pièce principale d'être un espace essentiellement domestique " (Les Sauniers d'Oléron..., op. cit., p. 97). 
ment, à vocation économique. Cette dernière renfermait deux fûts pour tout mobilier et une " essée ", sorte de pelle-fourche. Cet exemple de logement à deux pièces, typique du monde de la dépendance, offrait encore la possibilité d'une séparation des espaces que les habitants de pièces uniques, souvent proches de la misère, avaient du mal à reproduire. La demeure d'André Renesme ${ }^{18}$, pêcheur à l'Éguille, n'échappait pas à la polyvalence domestique de son unique chambre basse. Comme chez de nombreux sauniers $^{19}$, on rencontrait vis-à-vis du lit et de la table un outillage précieux quoique modeste : un "rêt appelé fayant pour la pêche aux meuils ", mais surtout " une cuve et dix fûts de chêne contenant une barrique ".

La pluriactivité était présente sous la forme de rares outils et de quelques fûts chez les plus humbles. Pour entreposer l'outillage, les stocks, et abriter le capital productif, d'autres espaces spécifiques, totalement professionnels ceux-là, complétaient le plan des maisons de gens de mer sortis de la misère.

\section{Le chai omniprésent}

Possesseurs des maisons les plus spacieuses et disposant des pièces de vie les plus spécialisées, les capitaines possédaient une grande diversité de dépendances et pièces annexes. Ils donnent une bonne idée de la diversité des activités productives qu'on recensait chez les marins. Au sein des 23 demeures " bourgeoises " visitées, le rapport aux activités agricoles se dessinait clairement. Propriétaires de maisons à étage, les capitaines de la Seudre disposaient de plusieurs greniers. Là, c'est par dizaines que les notaires prisaient des bouteilles de verre, stockées vides à l'étage; la garde du vin bouché était donc une pratique courante. Viticulture et vinification étaient en effet très largement répandues dans la bourgeoisie maritime. Cette capacité à conserver le vin était associée à l'une des dépendances les plus caractéristiques de la Saintonge littorale : le chai. Quinze maisons bourgeoises en possédaient au moins un et le "mobilier " des 21 chais recensés se limitait à la futaille (treuil "à fouler la vendange ", cuves, basses à vendange, barriques et fûts). Chez François Chambon, capitaine à Marennes ${ }^{20}$, " deux mauvaises basses " s'ajoutaient à " quatre cuves contenant 4 barriques, 8 fûts de barrique et 5 de tierçon ". Mobilisant à lui seul $30 \%$ des annexes, le vin était donc un élément majeur du quotidien de la bourgeoisie maritime en Saintonge.

18. Arch. dép. de Charente-Maritime, 3E LV/40, le 21 octobre 1762, par Me Dillé jeune, Saujon.

19. «Pour les plus démunis qui ne disposaient pas de pièce annexe, la chambre abritait une autre fonction : elle était alors le lieu où s'entassaient les outils et autres matériaux " (EDESA, Florence, Les Sauniers d'Oléron..., op. cit., p. 97).

20. Arch. dép. de Charente-Maritime, 3E XX/475, le 19 juin 1765, inventaire par $\mathrm{M}^{\mathrm{e}}$ Veillon aîné, Marennes. 
La possession d'un chai n'était pas un marqueur de la bourgeoisie tant il était courant sur tout le littoral saintongeais ${ }^{21}$. Le chai n'était pas non plus une marque d'appartenance au monde des marins, puisqu'on le rencontrait aussi chez les sauniers d'Oléron ${ }^{22}$. Dans les 32 foyers économiquement indépendants, pour moitié les dépendances visitées étaient consacrées au vin. Pas moins de 29 chais étaient recensés par les notaires. Constant, le poids des annexes consacrées au vin ne se démentait même pas chez les plus humbles. Ainsi, dans leurs 22 demeures, si la cave disparaissait totalement, le chai était rencontré à 14 reprises. En ajoutant le " cuvier " de Jean Palissier, cannonier à L'Éguille ${ }^{23}$, les annexes dédiées au vin représentaient $65 \%$ des 23 dépendances et annexes que les notaires visitaient.

Les gens de mer semblaient donc pleinement membres de la société littorale dont ils ne se distinguaient en rien par l'existence de pièces spécifiques à leurs occupations maritimes. Il existait bien des différences entre les catégories sociales mais elles se déclinaient en termes de diversité et de nombre d'annexes spécialisées.

\section{La boutique du matelot au long cours}

Dans le monde de l'indépendance économique, immédiatement après la viticulture et l'artisanat, le commerce était l'activité complémentaire des familles de gens de mer. Aussi pouvait-on recenser six " boutiques " : deux chez des maîtres de barque, trois chez des charpentiers et une à Riberou chez le tonnelier Pierre Gélineau ${ }^{24}$. Cette spécialité féminine marquait bien l'identité maritime, car contrairement au chai, elle n'était pas si courante dans les périphéries rurales d'Ancien Régime. Le commerce était aussi l'apanage des foyers ayant des revenus suffisants pour épargner et investir dans cette activité.

À côté de la chambre, pièce de vie partout présente, le chai était la dépendance caractéristique des demeures des gens de mer de la Seudre. Comme la plupart des Saintongeais maritimes, les gens de mer du Quartier de Marennes étaient souvent un peu vignerons et plus souvent encore, marchands de vin. L'organisation de leurs maisons s'en ressentait. S'il vivait dans une pièce unique, la futaille constituait l'environnement du marin; ailleurs, c'était une chambre qui était annexée par l'activité viticole. Le propriétaire d'un chai était déjà capable de séparer vie et travail, même si la vigne devait alors cohabiter avec d'autres outils, ceux du travail du bois ou de la pêche pour l'essentiel. Bourgeois, le propriétaire était en mesure de

21. Corinne Papot a en effet pu recenser 41 chais parmi 65 inventaires (Vivre à Marennes dans la deuxième moitié du XVIIf siècle, université de Poitiers, mémoire de maîtrise, 1997).

22. Florence Edesa indique ainsi que " chais, celliers et caves servaient à entreposer les futailles et les outils de vigneron " (Les Sauniers d'Oléron..., op. cit., p. 99),

23. Arch. dép. de Charente-Maritime, 3E LV/58, 15 mai 1781, inventaire par $\mathrm{M}^{\mathrm{e}}$ Dillé jeune, Saujon.

24. Arch. dép. de Charente-Maritime, 3 E LV/22, le 4 octobre 1745, inventaire par $\mathrm{M}^{\mathrm{e}}$ Perruchon, Saujon. 
spécialiser les espaces de sa maison : le chai, la cave pour la garde du vin, un local commercial loué à bail. Là étaient les marques de la bourgeoisie.

\section{Un capital productif extrêmement diversifié}

En tout point semblable à la maison saintongeaise, la demeure des gens de mer révélait la pluriactivité de ses habitants. Le chai était partout ou presque. Quant à la boutique, tenue par la femme de la maison, elle était bien plus caractéristique d'une pluriactivité " maritime ". Au-delà de l'organisation de la maison, c'est aussi par la détention d'un outillage et de stocks spécifiques, que l'exercice d'activités complémentaires peut être révélé.

\section{De la pauvreté à la subsistance}

Sous la barre des 700 livres tournois de biens, les 36 marins les plus modestes (marins, matelots, mariniers et pêcheurs) partageaient la condition des journaliers et sauniers. Rapporté à la modestie de leur patrimoine, le capital d'exploitation dépassait les $30 \%$. L'ensemble était cependant inégalement réparti et investi. Les seuls outils, engins de pêche et embarcations, représentaient $18 \%$ de l'inventaire moyen. C'est pourtant la rareté de tels investissements qui prévalait, seulement présent chez 5 marins sur 36 . De plus, la vétusté de ce capital lié à la navigation confirmait la dépendance de la plupart de ces individus, à l'égard des propriétaires de navires.

Les intérieurs révélaient cependant l'existence d'outils plus terriens qui permettent d'explorer la thématique de la pluriactivité. En effet, instruments agraires, outils liés à l'artisanat et cheptel représentaient $12 \%$ du patrimoine. Pourtant, les outils les plus communs chez le moindre journalier ${ }^{25}$ restaient rares chez les matelots. Un hachot et un coin à fendre, le tout estimé 4 livres tournois, chez le matelot Louis Pain ${ }^{26}$ de La Tremblade, 8 livres tournois consistant en 2 ferrées (pelle étroite à creuser les marais salants), 1 pelle et 3 serpes chez le tonnelier François Berton ${ }^{27}$ de Luzac ou encore une ferrée et une barre à planter la vigne chez le matelot André Lortie ${ }^{28}$, au Breuil-duPas, pour 6 livres tournois. Seuls dix inventaires mettent en avant la possession de ces outils qui rapprochaient leurs propriétaires de leurs voisins journaliers et autres manœuvriers. En second lieu, on est surpris de voir seulement trois des sept tonneliers posséder des outils ${ }^{29}$, le tout pour des

25. Jacques Péret rencontre fréquemment de modestes outils aratoires (piardes et besoches), le " dail " inséparable de son propriétaire lorsqu'il était occupé aux moissons, de " pauvres outils bricolés " afin de travailler dans les bois et à l'entretien des haies (Les Paysans de Gâtine poitevine au XVIII siècle, La Crèche, Geste Éditions, 1998, p. 141-142).

26. Arch. dép. de Charente-Maritime, 3E LXXII/231, 17 juillet 1782, Me Rousseau jeune, Arvert.

27. Arch. dép. de Charente-Maritime, 3E XX/619, 31 août 1798, Me Fontenelle, Marennes.

28. Arch. dép. de Charente-Maritime, 3E LV/27, 21 juin 1751, Me Dillé aîné, Saujon.

29. Caroline Bidon a aussi constaté ce phénomène pour nombre de matelots spécialisés, personnellement dépourvus d'un outillage qui était sans doute fourni à l'armement (Effets et hardes des gens morts en mer..., op. cit., p. 101). 
sommes modestes. Exemplaires, les 14 livres tournois d'outillage chez Daniel Séguineau ${ }^{30}$, tonnelier au Breuil-du-Pas, consistaient en une tenaille, un couteau à deux manches, une asse à rabattre le fer, une colombe (rabot) et une chaudière à bray. En l'absence de véritable pièce spécialisée (chai, atelier), on peut penser que dans cet outillage figurait une caisse à outils de matelot tonnelier, telle que peuvent la révéler les inventaires des gens morts en mer ${ }^{31}$.

En définitive, parmi les 36 marins disposant de moins de 700 livres tournois de biens, une grosse moitié disposait d'un capital professionnel inégal mais rares étaient ceux qui possédaient de quoi naviguer ou pêcher - j'en dénombre 5. Avec un gradient d'indices d'une production autonome, l'image d'un monde dépendant, puisque n'ayant souvent que ses seuls bras pour gagner sa vie, semblait bien s'imposer.

\section{De la subsistance à l'indépendance}

L'indépendance économique était acquise pour le groupe des 28 charpentiers et membres de la maistrance. Le poids du salariat était certainement responsable de la rareté de la propriété d'embarcations : trois. À la différence des esquifs des plus humbles, on avait affaire ici à des embarcations de transport, affectées au bornage ou au cabotage. La propriété d'une barque restait associée aux marchands et négociants, ce qui différait fortement de la copropriété observée au XVIII ${ }^{\mathrm{e}}$ siècle dans le Morbihan, avec des sociétés où les marins étaient partie prenante ${ }^{32}$.

En revanche, 10 inventaires sur 28 révélaient l'existence d'un capital à vocation agricole. Les outils des plus humbles étaient là, mais ils étaient plus nombreux. Ferrée, pelle, bedochon, pioche, fourche, aucun des outils ne valait plus d'une livre pièce, et chaque marin n'en possédait pas plus de 4 à 5 différents. De plus, le capital mobilisé dans du matériel viticole était significatif. Six inventaires rapportaient l'existence de fûts, la plupart de chêne et plutôt bons. Les charpentiers en avaient l'exclusivité, mais rien ne dit qu'ils ne stockaient pas le vin de leurs voisins. On est frappé par l'absence de treuil (pressoir) ou de basses (futailles de vendange) et la rareté des cuves (pour la fermentation) dont seuls Jean Lortie ${ }^{33}$ au Breuil du Pas et Samuel Fonteneau ${ }^{34}$ à La Tremblade étaient propriétaires. Chez les salariés du long cours, la présence à terre - à l'inverse des pêcheurs ou

30. Arch. dép. de Charente-Maritime, 3E LV/31, 3 avril 1755, Me Dillé aîné, Saujon.

31. Dans la partie 2 de son mémoire, qu'elle a consacré à la vie professionnelle, Caroline Bidon donne une liste semblable d'outils retrouvés dans les affaires personnelles de matelots tonneliers (Effets et hardes des gens morts en mer..., op. cit., p. 110-111).

32. Benoît DeFEe, Caboteurs et cabotage du quartier du Port-Louis (1709-1756), université de Bretagne-Sud, mémoire de maîtrise, sous la direction de Gérard Le Bouëdec, 1997, p. 89.

33. Arch. dép. de Charente-Maritime, 3E LV/48, le 28 août 1771, par M ${ }^{\mathrm{e}}$ Dillé jeune, Saujon.

34. Arch. dép. de Charente-Maritime, 3E LXXI/147, le 14 sept. 1774, par M ${ }^{\mathrm{e}}$ Rousseau jeune, Arvert. 
des caboteurs ${ }^{35}$ - ne coïncidait pas avec les saisons végétatives. Les uns prêtaient peut-être leur capital à d'autres, en échange de services (journées de travail, surveillance des récoltes, etc.). La configuration des villages avec des " chais, appartenant à des propriétaires différents, par endroits contigus et formant de petites zones d'activité spécialisée ${ }^{36}$ " plaide en faveur de cette thèse.

Quatorze individus possédaient des réserves agricoles. Chez quatre d'entre eux, il s'agissait de "bleds " mais aussi de ces " fèves et gesses " récoltées sur les bosses de marais; pour une dizaine d'autres, le vin constituait l'essentiel des réserves. Les disparités étaient grandes, entre les 188 fûts de vin stocké dans les 12 barriques du chai de Pierre Lamoureux ${ }^{37}$, charpentier au Lindron (Marennes) et l'unique barrique de " boisson blanche " prisée 1 livre tournois chez Julien Biron ${ }^{38}$ charpentier à Peudrit (Saujon). Par ailleurs, huit actes d'acquisition sur onze recensés concernaient des vignes ${ }^{39}$. Débordant du cadre de la subsistance, les stocks de vin étaient associés à une activité commerciale comme le suggéraient les quantités conservées : chez le charpentier Étienne Sauvignon ${ }^{40}$, dont l'épouse Marie Garnier tenait boutique à La Boirie (Marennes), quatre tonneaux prisés au chai contenaient du vin " actuellement débité, prix dû par ceux qui l'ont acheté" ".

L'activité de boutique ou de commerce faisait entrer la balance avec son assortiment de poids en plomb dans quatre inventaires. Si la boutique de Marie Dexmier épouse Gaborit ${ }^{41}$, charpentier à Brouage, contenait pour 347 livres tournois de marchandise, le stock était souvent plus modeste, de 30 à 50 livres tournois la plupart du temps. Les articles vendus illustraient la double spécificité des gens de mer : hommes de la terre et du grand com-

35. Jean-François Henry indique par exemple que seuls $6 \%$ des campagnes de cabotage prenaient place en été, laissant la main d'œuvre maritime disponible pour les moissons, même si la pêche côtière fournissait alors une activité de relais (Des marins au siècle du Roi-Soleil, l'île d'Yeu sous le règne de Louis XIV, thèse de $3^{\mathrm{e}}$ cycle, Janzé, éd. Y. Salmon, 1982).

36. Étant donné la similitude des habitats oléronnais et de la Seudre, cette remarque extraite de L'T̂le d'Oléron. Inventaire général des monuments et des richesses artistiques de la France, éd. Geneviève Renaud-Romieux, Jean-Pierre Roussel, Poitiers, Connaissance et promotion du patrimoine de Poitou-Charentes, 1993, p. 54, semble transposable aux pays d'Arvert ou de Marennes.

37. Arch. dép. de Charente-Maritime, 3E LXI/340, le 13 mai 1799, par M $^{\mathrm{e}}$ Bourdain, Marennes.

38. Arch. dép. de Charente-Maritime, 3E LV/57, le 14 janv. 1780, par Me Dillé jeune, Saujon.

39. Dans l'île d'Oléron voisine, "l'artisanat profitait des temps de crise pour se constituer un foncier propre à fournir un complément de ressources ", selon Raymond JousMET, "Le marché foncier de l'île d'Oléron (1750-1786) ", Écrits d'Ouest, Cahiers Rochelais d'Histoire Régionale, $\mathrm{n}^{\circ}$ 3, 1994.

40. Arch. dép. de Charente-Maritime, 3E XX/591, le 5 janv. 1770, par Me Fontenelle, Marennes.

41. Arch. dép. de Charente-Maritime, 3E XX/593, le 24 février 1772, par Me Fontenelle, Marennes. 
merce. Ainsi, le tiers de la marchandise proposée à la vente était-il constitué de produits tropicaux : tabac, café, thé, sucre, girofle, poivre et muscade. Ne s'agissait-il pas là de la pacotille qu'on tolérait à bord? Il ne faut pas négliger l'existence de circuits plus réguliers. Geneviève Normand ${ }^{42}$, épouse Dubois, et Anne Jollivet ${ }^{43}$, épouse Husseau, (deux charpentiers de Riberou), n'étaient-elles pas " englouties dans la rivière de Bordeaux dans la nuit du $1^{\text {er }}$ au 2 nivose an III, alors qu'elles allaient s'approvisionner à Bordeaux "? En dehors de ces produits des Isles, véritable spécificité du monde de la mer, la boutique proposait à la vente de la mercerie (fil, laine, coupons de tissu), de la droguerie (balais, sabots, chandelles), de la quincaillerie (clous, cordes, crochets) et quelques denrées (beurre, fromage).

Charpentiers et maîtres s'adonnaient donc à l'agriculture et au commerce. Appartenant au monde de l'artisanat, 13 de ces marins détenaient aussi des outils et des stocks spécifiques. Trois charpentiers semblaient avoir développé une activité d'atelier, souvent localisée dans un chai. Jean Gaborit présentait un outillage lourd et important : " établi, tarrières à percer les poutres, vrilles, varloppes, ciseaux et bouvets " mais aussi " deux échelles à rolons et un palan à deux poulies ", le tout pour 230 livres tournois. Quant à Gabriel Griffon ${ }^{44}$, charpentier à La Motte (Marennes), il possédait " hache, herminette, ciseaux, marteau, scies " dans son chai où l'on trouvait aussi " un banc de taillage et son couteau à deux manches, une masse de tonnelier et une chaudière à bray ". Cet artisan était polyvalent, comme l'étaient Samuel Fonteneau à La Tremblade ou Pierre Lamoureux au Lindron. Tous disposaient d'une "brouette ", à l'aide de laquelle ils transportaient leurs outils à pied d'œuvre. En marge, la masse des matelotscharpentiers disposait d'un outillage " portatif ", stocké dans un grenier ou une chambre, à l'image de la caisse à outils des tonneliers. Ils étaient pourtant nombreux (huit) à ne pas avoir d'outils, dépendant alors de l'armateur qui les enrôlait.

Ainsi, les deux tiers de cette catégorie de gens de mer alliaient à la navigation des activités complémentaires susceptibles d'assurer des compléments de revenus ou de fournir le moyen d'une éventuelle reconversion. Ces gens de mer les plus qualifiés possédaient finalement peu d'embarcations, de matériel nautique ou de pêche. Le fait est que la forte mobilité sociale de père à fils faisait souvent du marin de la Seudre un parent du paysan ou du vigneron voisin. Débarqué des longs courriers, le charpentier ou l'officier marinier se tournait plus volontiers vers la terre que vers la mer pour compléter ses revenus. Titulaires d'un savoir-faire, les charpentiers possédaient majoritairement des outils, même s'ils étaient peu nombreux

42. Arch. dép. de Charente-Maritime, 3E LV/76, le 13 janvier 1795, par Me Perruchon, Saujon.

43. Arch. dép. de Charente-Maritime, 3E LV/76, le 26 mars. 1795, par Me Perruchon, Saujon.

44. Arch. dép. de Charente-Maritime, 3E XX/615, le 8 mai 1794, par Me Fontenelle, Marennes. 
à s'être fixés à l'atelier, au chai le plus souvent. La vigne semblait être le dénominateur le plus commun de ces marins. À défaut d'investir dans le vin, on tenait parfois boutique. Les épouses, modestes femmes d'affaires, proposaient aux chalands les produits des Isles comme les objets domestiques les plus communs.

\section{Un capital important et diversifié : un gage de sécurité?}

Avec leur activité tournée vers le long cours, on est d'emblée surpris de rencontrer chez quelques capitaines, la propriété de rares et modestes embarcations, "sur leurs amarres " en Seudre. On peut émettre l'hypothèse d'usages très variés, allant d'une modeste entreprise à la récréation.

Le recensement de l'outillage artisanal révélait neuf inventaires dont l'essentiel était tourné vers le travail du bois. Il semble que, de la menuiserie au chantier naval en passant par la tonnellerie, la palette des métiers de la filière bois constituait une des bases culturelles du monde de la mer en Seudre. Y compris chez les plus riches, comme chez Jean You à Marennes, le chai ou le grenier abritait la traditionnelle herminette et ses variantes (hache, hachot, hachereau, serpe) mais aussi le marteau, le ciseau, le rabot, la scie. Jacques Papin ${ }^{45}$ de La Tremblade possédait en plus « un palan, deux échelles et une brouette ", le parfait équipement du charpentier que l'on trouvait aussi chez Daniel Cerclé ${ }^{46}$ au Moulin du Piochet (Arvert), chez Elie Suidre ${ }^{47}$ Grand'Rüe à Marennes ou chez Jean Husseau de Riberou. Mobilisant de 5 à 50 livres tournois suivant les inventaires, cet outillage maintenait sans doute une tradition du pays. Il expliquait aussi la mobilité professionnelle de père en fils entre les mondes du chantier naval et des capitaines. D'autant qu'il ne s'agissait nullement d'un capital dormant, comme l'attestait la présence de stocks de bois d'œuvre. Ce travail du bois n'était pas étranger à la propriété d'une futaille importante liée à la culture de la vigne et au commerce du vin.

Sur les 21 inventaires instrumentés, 12 signalaient la présence de stocks viticoles, en quantité marchande pour la plupart. Henry Girard, à Saujon, était ainsi propriétaire de 70 livres tournois de vin rouge stocké dans les quatre barriques de son sellier, Louis Guillocheau possédait trois barriques de " vin rouge de la récolte dernière ", pour un montant de 50 livres tournois. Quant aux capitaines Papin (La Tremblade), Cerclé (Chaillevette) ou Chevrou (Le Chapus), ils possédaient plus de 10 barriques tant de vin rouge que de blanc, un stock estimé entre 150 et 300 livres tournois chacun. Il y avait là une marque d'intégration des gens de mer à l'économie

45. Arch. dép. de Charente-Maritime, 3E LXXII/248, le 3 décembre 1801, par Me Rousseau jeune, Arvert.

46. Arch. dép. de Charente-Maritime, 3E LXXII/248, le 24 janvier 1807, par Me Rousseau jeune, Arvert.

47. Arch. dép. de Charente-Maritime, 3E XX/377, le 16 décembre 1782, par Me Durand, Marennes. 
locale, puisque les vins stockés étaient la plupart du temps " du païs " et que la propriété foncière était très importante. Entre sel, vin, blé et bétail, les choix d'investissement en Saintonge maritime étaient assez larges. La première propriété par ordre d'importance était détenue par le saujonnais Henry Girard : deux " mestairies ", l'une au nord de Saujon au lieudit Sommiers acquise pour 6000 livres tournois et l'autre au sud, près de Semussac, appelée la Gondonnière et d'une valeur de 4700 livres tournois. Le faire-valoir indirect était de mise, la rentabilité d'un placement, l'objectif ultime : on était loin de la pluriactivité.

Au total, dans le groupe des capitaines de navires, les investissements productifs allaient vers l'artisanat, le commerce et la terre. Révélatrice d'une culture maritime particulière à la Seudre, la possession de l'outillage artisanal était la marque d'un héritage matériel dont on se transmettait le savoir-faire. Le travail du bois semblait indissociable de l'entretien de barriques et autres fûts destinés au stockage du vin. Les capitaines s'intégraient à une société littorale dont le travail de la vigne transcendait les clivages.

L'étude des maisons et du capital d'exploitation des gens de mer offre un regard sur la pluriactivité des salariés du grand commerce au XVIII siècle. Le salariat expliquait sans doute la rareté du capital "maritime " (barque, agrès, engins de pêche). À terre, ce sont trois types d'activité qui étaient repérables. La plus rare, mais non la moins originale, était assumée par la femme, qui s'adonnait à un petit commerce alimenté soit par la "pacotille " du mari, soit par des fournisseurs auprès desquels elle se rendait, en ville. Lorsque l'époux était en mer (les deux tiers du temps ${ }^{48}$ ), la famille pouvait être mobilisée dans des travaux agricoles, centrés sur la culture de la vigne et la vinification. Enfin, pour entretenir la futaille mais aussi proposer ses services "à la tâche ", l'homme qui séjournait chez lui avait à sa disposition un outillage, allant de la simple caisse à l'atelier aménagé.

Chez les matelots, l'absence de spécialisation rimait aussi avec l'absence d'outils. On était pauvre et dépendant du seul salaire, ce qui était parfois pire que la condition des journaliers qui disposaient tout de même de quelques outils agricoles. Avec la maîtrise d'un savoir-faire (pêcheur, tonnelier), venait, en même temps qu'une fortune un peu moins misérable, la possession d'outils et de stocks qui pouvaient constituer l'essentiel de la fortune. Poser quelques filets, draguer les fonds à la recherche de quelques coquillages pour ceux-ci, proposer ses services aux vignerons ou travailler au port avant de s'embarquer par intermittence pour ceux-là, telles étaient les activités quotidiennes qui permettaient, sinon de rompre avec la dépendance, au moins de s'en dégager, les bonnes années. Pêcheurs et tonneliers

48. Étude des rythmes de travail dans notre thèse, Les marins de la Seudre (XVIII XIXe siècle). Du sel charentais au sucre antillais, La Crèche, Geste Éditions, mai 2005. 
étaient aux marges des mondes de la dépendance et de l'indépendance, comme les artisans et les sauniers les plus aisés.

Parce qu'ils pouvaient davantage s'appuyer sur leur savoir-faire (charpentiers, maîtres au cabotage) ou sur leur expérience (officiers mariniers), des gens de mer plus fortunés se distinguaient par la possession d'un capital productif plus diversifié (outillage, chai, boutique, terres) qu'ils exploitaient directement ou en famille et dont ils tiraient des revenus complémentaires à l'activité salariée. La pluriactivité dont ils faisaient preuve les mettait finalement à l'abri des coups durs, en leur ouvrant la porte de toutes les reconversions (agriculture, commerce, pêche, transport, artisanat) : ils figuraient en Seudre le monde de l'indépendance économique, au même titre que les laboureurs de l'Aunis voisine.

Au sommet de la hiérarchie sociale, les capitaines de navire se fondaient dans la bourgeoisie, moins par la nature de leur fortune que par leurs investissements fonciers pourvoyeurs de rente foncière. Par ailleurs, l'obstination avec laquelle les capitaines de la Seudre conservaient un outillage destiné à travailler le bois n'est pas sans interroger l'historien. Sur 85 inventaires étudiés, 54 propriétaires d'outils de menuiserie, tonnellerie ou charpentage peuvent être recensés. Si la possession d'un capital viticole intégrait les gens de mer à la société littorale saintongeaise, il semble néanmoins que les marins s'étaient fait une spécialité du travail du bois. 


\section{RÉSUMÉ}

À la fin du XVIII ${ }^{\mathrm{e}}$ siècle, un millier de gens de mer habitent autour de l'estuaire de la Seudre, une zone rurale et maritime. Ils travaillent comme salariés du grand commerce, à La Rochelle et Bordeaux mais passent souvent plus de la moitié de leur vie à terre. C'est grâce à l'étude des archives des notaires que l'on peut étudier leur mode de vie, a travers l'organisation de leurs maisons, la nature de leurs stocks et de l'outillage qu'ils possèdent. Les activités de second plan suivent la hiérarchie sociale. Les plus pauvres pratiquent la pêche côtière, les plus riches exploitent des terres, les catégories intermédiaires font du commerce. Pour tous, la diversification de l'activité passe par deux marqueurs principaux : l'exploitation de la vigne et le travail du bois. Quel que soit leur niveau social, tous les marins de la Seudre sont concernés, ce qui constitue une particularité de ce monde maritime dans la France de la fin du $\mathrm{XVIII}^{\mathrm{e}}$ siècle.

\section{ABSTRACT}

At the end of the eighteenth century, a thousand seamen lived close to the estuary of the river Seudre, a rural and maritime area. They worked as sailors for the long-distance trade based in Bordeaux or La Rochelle, but they also spent over half of their time on land. Thanks to the archives of the notaries, it is possible to study their way of life, from the way they organised their houses to the nature of their goods and stocks and the tools that they owned. Their place in the social hierarchy seems to have dictated the nature of the other work they undertook. The poorest engaged in coastal fishing whilst the more wealthy farmed their land. Those in-between often had a little shop at home. But two activities were shared by all social categories: winemaking and woodworking. This seems to be particular to this maritime society of early modern France. 